\title{
Integrating a Binaural Beat into the Soundscape for the Alleviation of Feelings
}

\author{
Noko Kuratomo ${ }^{1}$, Yuichi Mashiba ${ }^{1}$, Keiichi Zempo ${ }^{1}$, Koichi Mizutani ${ }^{1}$, and \\ Naoto Wakatsuki ${ }^{1}$ \\ University of Tsukuba, 1-1-1, Tennodai, Tsukuba, Ibaraki 305-8573, Japan \\ zempo@iit.tsukuba.ac.jp
}

\begin{abstract}
A binaural beat is the sound that makes beat in human brain by providing sounds of different frequencies to left and right ears. It has been reported that the brain wave of frequency difference is easily induced when we listen to it. In this study, it is verified whether brain waves can be induced if a binaural beat is provided in a living environment. In reproducing living environments, soundscape is used and binaural beat is integrated into it. Brain wave is measured and users' subjective assessment is conducted in experimental. As a result, the effect of binaural beat to the alleviation of feelings is verified. It has been clarified binaural beat has positive effects, even under negative condition.
\end{abstract}

Keywords: Binaural Beat, User Interface

\section{Introduction}

We live in a variety of environments and feel happy, relaxed, stressed and so on. Positive emotion is increased in nature [4], while negative emotion is increased in urban environments featuring sounds like car horns and ambulance sirens, as well as in situations in which we are confined, like while studying $[5,6]$. So, if emotion can be controlled to suit the situation, we can live with better conditions, e.g. amplifying positive emotions and alleviating negative emotions, as shown in Fig. 1.

It is known that human emotions are related to brain waves [11]. Brain wave activity is observed by measuring its frequency as electric signals. Brain wave signals include delta $(0.1$ to $3.5 \mathrm{~Hz})$, theta $(3.5$ to $7.5 \mathrm{~Hz})$, alpha $(7.5$ to $13 \mathrm{~Hz})$, beta $(13$ to $30 \mathrm{~Hz})$, and gamma $(30$ to $50 \mathrm{~Hz})$ [12]. The conditions connected to these brain wave types are shown in Table 1 .

It is reported that the brain wave condition is induced by external stimuli [1]. When a flash is used as a stimulus, there is a possibility of giving users become acute symptoms based on photosensitivity $[2,3,8]$. On the other hand, it is hard to induce the desired condition by the sound since the ranges are different between brain waves and audible frequencies. Therefore, a binaural beat is proposed as a method of changing emotions.

A binaural beat is a sound that makes a beat in the human brain by playing sounds of different frequencies in the left and right ears respectively. An image 


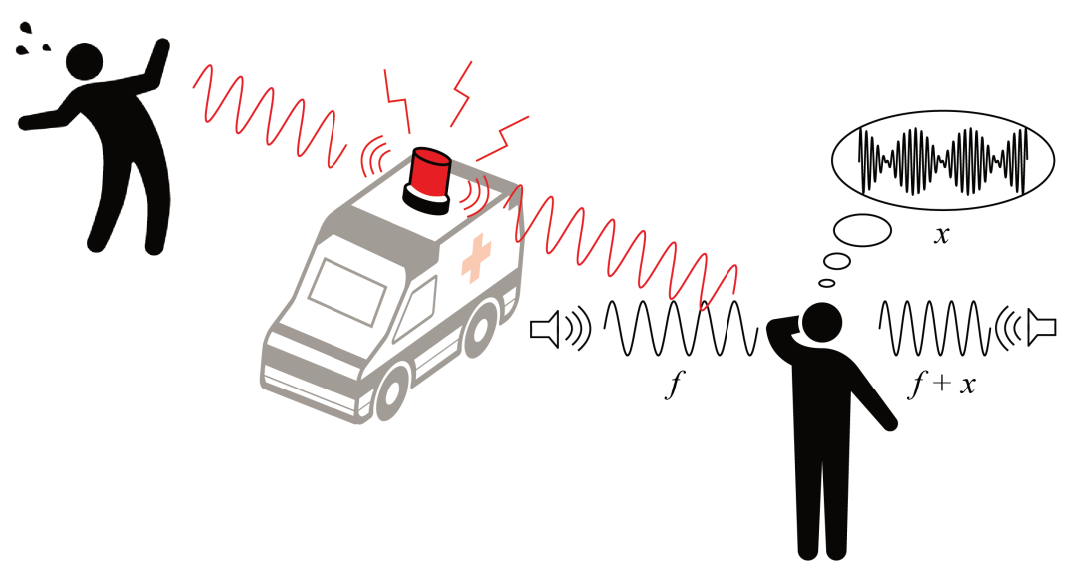

Fig. 1. Construction of a binaural beat and its assumed effect under negative emotion: If sounds of $f$ are provided at the left ear and sounds of $f+x$ are provided at the right ear, a sound of $x$ is recognized as a beat.

Table 1. Brain wave types and conditions [13]

\begin{tabular}{l|l|l}
\hline Type & Frequency & Conditions \\
\hline \hline delta & $0.1-3.5 \mathrm{~Hz}$ & deep sleep \\
\hline theta & $3.5-7.5 \mathrm{~Hz}$ & light sleep, creativity, insight \\
\hline alpha & $7.5-13 \mathrm{~Hz}$ & a calm and peaceful, yet alert state \\
\hline beta & $13-30 \mathrm{~Hz}$ & thinking, focused state, intensity or anxiety \\
\hline gamma & $30-50 \mathrm{~Hz}$ & perception, fear, consciousness \\
\hline
\end{tabular}

representing this concept is shown in Fig. 1. It is reported that a binaural beat can change the frequency of brain waves to a roughly desired state. For example, if sounds at $440 \mathrm{~Hz}$ are supplied to the left ear and sounds at $450 \mathrm{~Hz}$ supplied to the right ear, the frequency difference between ears is $10 \mathrm{~Hz}$, so an alpha wave is induced in the listener's brain and the listener may feel relaxed [7]. The desired brain wave condition determines which audio signals should be input.

So far, most research about the effects of binaural beats investigates a binaural beat used alone [7,10]. However, binaural beats should be applied to our daily life, and the effect of binaural beats on human brain wave activity in a real-life environment is still not clear.

Sound plays an important role in our living environment, which affects feelings. Sound environments created by nature and social activity are generally called soundscapes. Soundscapes are classified into six main types by Murray Schafer's soundscape taxonomy, shown in Table 2 [13]. Therefore, this soundscape taxonomy is able to be used for reproducing daily life in terms of sound. 
Table 2. Sounds of the environment categories [9]

\begin{tabular}{l|l}
\hline Category & Soundscape \\
\hline \hline Natural sounds & birds, chickens, rain, sea shore \\
\hline Human sounds & laughing, whispering, shouting, talking, coughing \\
\hline Sounds and society & parties, concerts, grocery stores \\
\hline Mechanical sounds & engines, cars, air conditioners \\
\hline Quiet and silence & wild space, silent forest \\
\hline Sound as indicators & clocks, doorbells, sirens \\
\hline
\end{tabular}

The purpose of this paper is to investigate a method to use a binaural beat to adapt oneself to a condition needed in a particular environment. When the integrated sound of the soundscape and the binaural beat is provided, the condition variable of the listener's brain wave is observed and examined.

\section{The intention of a binaural beat display}

To know how to control emotions by listening to a binaural beat in daily life, it is necessary to research how binaural beats affect brain activities in various environments. Users perceive a binaural beat as a beat made by two pure sounds. For example, when a stereo sound having a frequency difference corresponding to an alpha wave is input, a sound of that frequency is recognized as a beat. That is, to induce a desired brain wave condition, the signal which should be input is specified according to Table 1.

In experiments, daily environments are reproduced by using environmental sounds. Whether emotions change is analyzed through brain wave measurements and subjective assessment when binaural beats are integrated into soundscapes, e.g., distracting places or annoying scenes.

\section{Experiment}

\subsection{Binaural sound signals}

To verify the possibility of inducing feelings, three kinds of binaural beat corresponding to three kinds of brain waves are prepared. The frequency difference of the sound is set at 5,12, and $23 \mathrm{~Hz}$ corresponding to theta, alpha, and beta waves, respectively. In this experiment, we set a sine wave of $440 \mathrm{~Hz}$ as the standard, which is provided to the left ear. Tuned up sine signals at 445, 452, and $463 \mathrm{~Hz}$ were provided to participants' right ears.

Soundscapes are classified into six types by Murray Schafer's soundscape taxonomy [9]. From this taxonomy, we prepared four kinds of the environmental sounds as outside stimuli to affect human feelings: sea, forest, shopping mall and ambulance siren. 


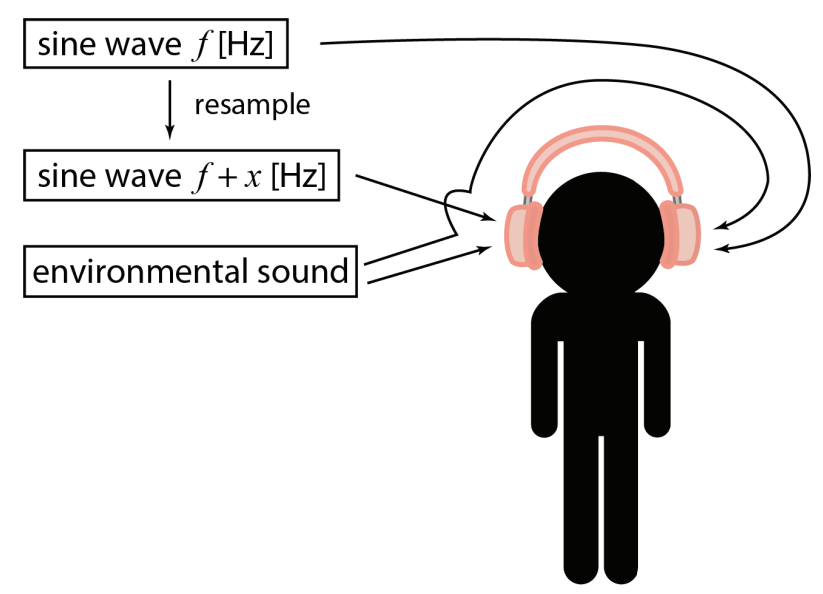

Fig. 2. The sound algorithm used in the experiment, containing a binaural beat integrated into environmental sound.

The sound algorithm is shown in Fig. 2. We set the ratio of the binaural beat to the soundscape to 1:9. A total of 12 kinds of experimental stimuli is prepared by integrating the three binaural beats into four soundscapes.

\subsection{Measurement equipment}

Participants wear three electrodes to measure brain waves and headphones to listen to the sound. Two electrodes are attached at the back of the head and one is at the earlobe. A photo of the experiment is shown in Fig. 3. We analyze the measured brain wave with the Fourier spectrum and examine the strength of each frequency component. For brain wave analysis, we use the data processing module developed by the Brain Function Research Center.

\subsection{Experimental protocol}

During the experiment, participants closed their eyes and moved as little as possible in order to minimize the influence of eye movements on brain wave measurement. The measurement time is $180 \mathrm{~s}$ per set. The flow of these experiments is shown in Fig. 4.

Sixteen sets (12 integrated sounds and four environment-only sounds) of experiments are carried out. Besides brain waves, participants assess the soundscape subjectively on two scales: upset to relax and uncomfortable to comfortable. There are seven assessment values, which are shown in Fig. 5. Participants consist of 10 people (average age: 23, 7 men and 3 women) with good hearing.

This experiment was reviewed and approved by the ethics committee of the organization to which we belong. 


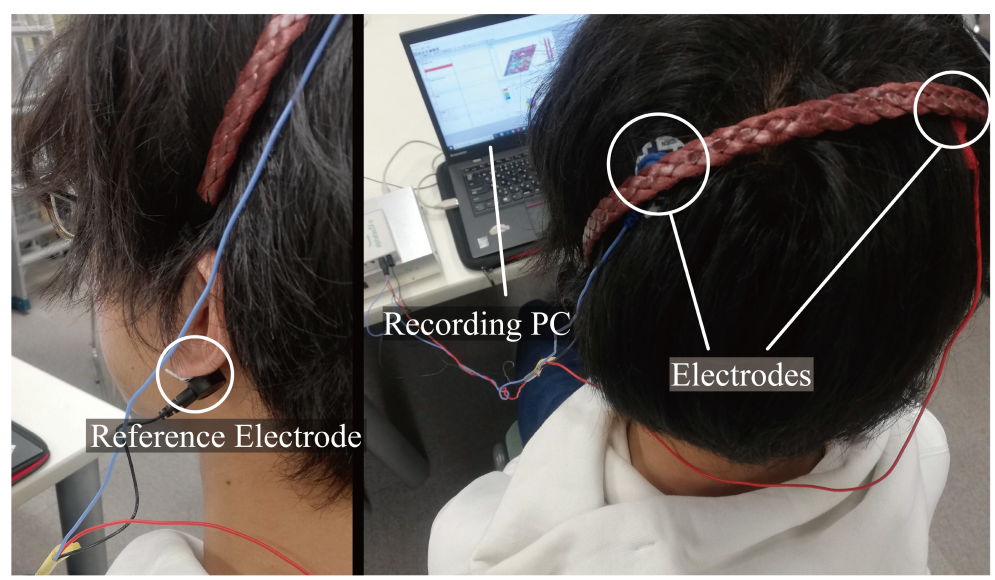

Fig. 3. Equipment for measuring brain waves

$\underbrace{\text { silent }}_{0: 00} \underbrace{\text { playing sound }}_{0: 30} \underbrace{\text { silent }}_{2: 30}$

Fig. 4. Flow of experiment

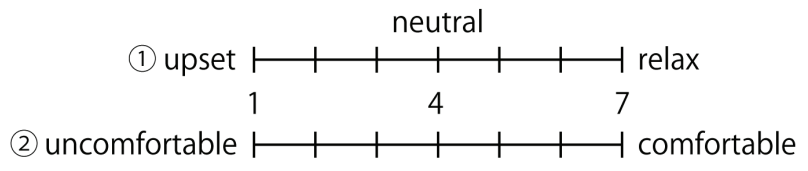

Fig. 5. Participant assessment axis

\section{Results and discussion}

Fig. 6 shows results in two ways: as a fluctuation of brain waves and as a participants' subjective assessments. The fluctuation of brain waves measured during the period from 60 to $120 \mathrm{~s}$ is analyzed, where the brain wave is stable. We measure the ratio of participants whose brain waves fluctuate as intended when the each sound is provided, compared to when listening to an environment-only sound. That is, it counts if the component of the theta wave increased when an integrated sound at $5 \mathrm{~Hz}$ frequency difference was provided. The same is true for both the alpha wave and $12 \mathrm{~Hz}$ frequency difference, the beta wave and $23 \mathrm{~Hz}$ frequency difference. In Fig. 6, the environmental sounds used in the experiment are sorted into (a)-(d). From this figure, the following findings arise. 


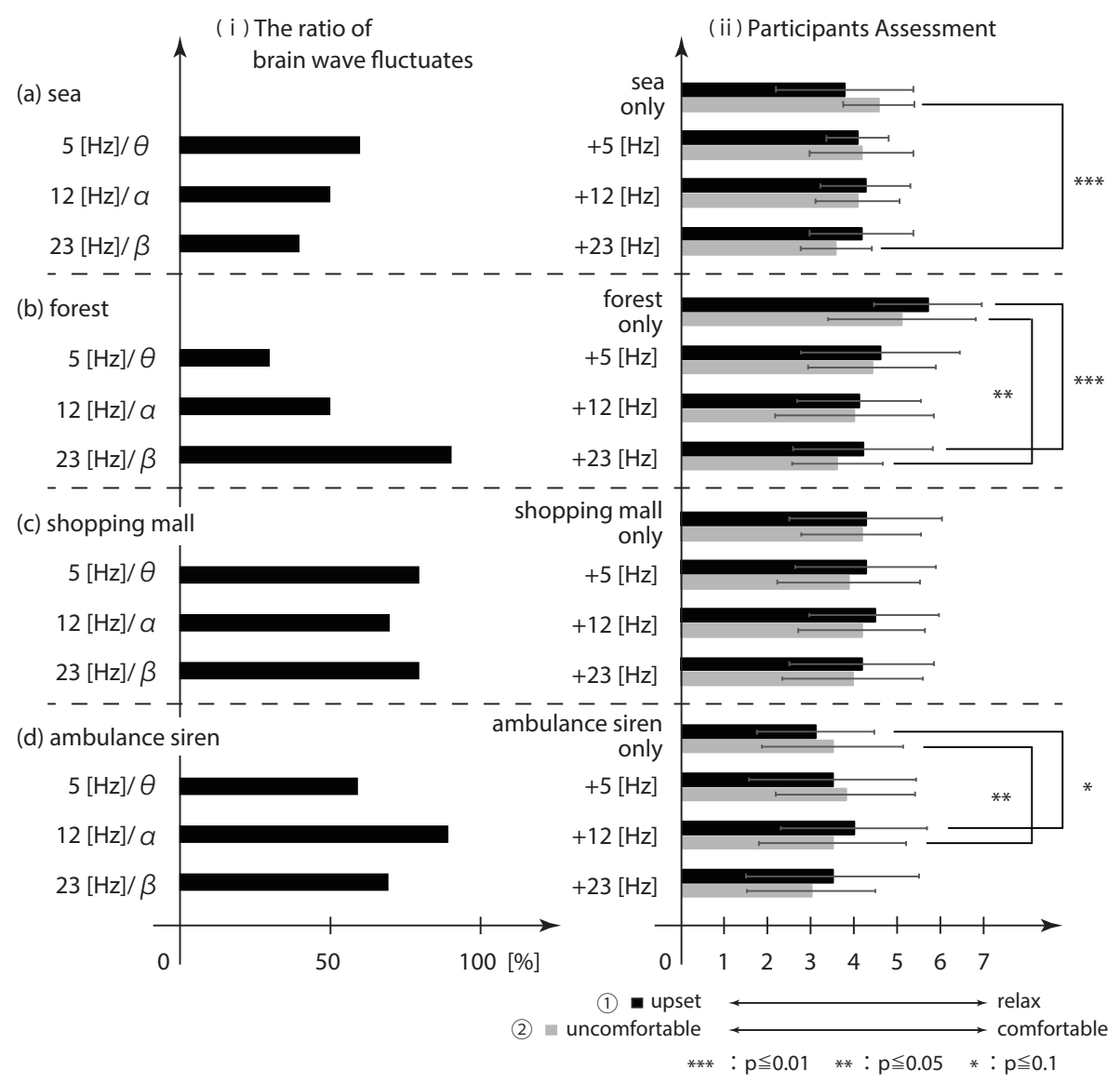

Fig. 6. Experiment Results - (i) shows the ratio of brain wave fluctuates and (ii) shows participants' assessment average and its standard deviation.

(1) According to (b)-(ii), most participants relaxed when in an environment of forest sound only. However, they became somewhat upset when sounds at a $23 \mathrm{~Hz}$ frequency difference were provided. As shown in (b)-(i), nine participants' beta waves increased. Therefore, the beta wave is likely induced in relaxed conditions.

(2) According to (d)-(ii), the ambulance siren makes participants feel upset and uncomfortable. Conversely, the subjective assessment improves when sounds at a $12 \mathrm{~Hz}$ frequency difference are provided. The ratio of brain waves in which the alpha wave increased is higher than others ((d)-(i)). It is clear that sounds at a $12 \mathrm{~Hz}$ frequency difference can alleviate distressing emotions.

These results can be also seen in the p-value in Fig. 6 (the p-value is calculated by a one-sided test only in cases in which the brain wave fluctuated as intended). 
However, it is hard to detect an improvement when participants are in a relaxed environment, and vice versa.

\section{Conclusion}

This study's purpose is to research whether emotions can be controlled by listening to a binaural beat in daily life. In the experiment, we investigated how a binaural beat integrated into the soundscape affects brain activities by measuring brain waves and collecting subjective assessments. In result, the effect of binaural beat integrated into soundscape to the alleviation of feelings is verified. It has been clarified binaural beat has positive effects, even under negative condition.

From now on, it is necessary to conduct experiments focusing on alleviating of feelings to more people. As areas for further study, the effects of a binaural beat on a person performing simple work should be investigated, and the possibility of applying a binaural beat to many situations in which someone may feel stressed.

\section{References}

1. Adrian, E.D., Matthews, B.H.: The berger rhythm: potential changes from the occipital lobes in man. Brain 57(4), 355-385 (1934)

2. Enoki, H., Akiyama, T., Hattori, J., Oka, E.: Photosensitive fits elicited by tv animation: an electroencephalographic study. Pediatrics International 40(6), 626630 (1998)

3. Fylan, F., Harding, G.: The effect of television frame rate on eeg abnormalities in photosensitive and pattern-sensitive epilepsy. Epilepsia 38(10), 1124-1131 (1997)

4. Hartig, T., Evans, G.W., Jamner, L.D., Davis, D.S., Gärling, T.: Tracking restoration in natural and urban field settings. Journal of environmental psychology 23(2), 109-123 (2003)

5. Ishikawa, T., Tatsumoto, M., Maki, K., Mitsui, M., Hasegawa, H., Hirata, K.: Identification of everyday sounds perceived as noise by migraine patients. Internal Medicine pp. 2206-18 (2019)

6. Jena, S.K., Misra, A.K., Mohanty, A., et al.: Effect of examination stress on blood sugar in medical students. CHRISMED Journal of Health and Research 3(4), 268 (2016)

7. Puzi, N.M., Jailani, R., Norhazman, H., Zaini, N.M.: Alpha and beta brainwave characteristics to binaural beat treatment. In: 2013 IEEE 9th International Colloquium on Signal Processing and its Applications. pp. 344-348. IEEE (2013)

8. Ricci, S., Vigevano, F., Manfredi, M., Trenité, D.G.K.N.: Epilepsy provoked by television and video games, safety of 100-hz screens. Neurology 50(3), 790-793 (1998)

9. Schafer, R.M.: The soundscape: Our sonic environment and the tuning of the world. Simon and Schuster (1993)

10. Settapat, S., Ohkura, M.: An alpha-wave-based binaural beat sound control system using fuzzy logic and autoregressive forecasting model. In: 2008 SICE Annual Conference. pp. 109-114. IEEE (2008) 
11. Stastny, J., Sovka, P., Stancak, A.: Eeg signal classification. In: 2001 Conference Proceedings of the 23rd Annual International Conference of the IEEE Engineering in Medicine and Biology Society. vol. 2, pp. 2020-2023. IEEE (2001)

12. Trivedi, P., Bhargava, N.: Effect of left and right hemisphere of brain in both eye open and close state on minimum power values and frequency of alpha wave activity. brain $\mathbf{6}(2)(2017)$

13. Zaini, N., Omar, H., Latip, M.F.A.: Semantic-based bayesian network to determine correlation between binaural-beats features and entrainment effects. In: Computer Applications and Industrial Electronics (ICCAIE), 2011 IEEE International Conference on. pp. 574-579. IEEE (2011) 